\title{
Affirmer l'identité du territoire : Une démarche de valorisation du littoral au Pays Marennes-Oléron
} Jean-Baptiste Bonnin, Yannick Bruxelle et Christophe Pelaprat

\author{
(2) OpenEdition \\ Journals \\ Édition électronique \\ URL : http://journals.openedition.org/ere/1069 \\ DOI : $10.4000 /$ ere.1069 \\ ISSN : 2561-2271 \\ Éditeur \\ Centr'ERE
}

Référence électronique

Jean-Baptiste Bonnin, Yannick Bruxelle et Christophe Pelaprat, « Affirmer l'identité du territoire : Une démarche de valorisation du littoral au Pays Marennes-Oléron », Éducation relative à l'environnement [En ligne], Volume 10 | 2012, mis en ligne le 20 décembre 2012, consulté le 21 février 2020. URL : http:// journals.openedition.org/ere/1069; DOI : 10.4000/ere.1069 


\title{
Affirmer l'identité du territoire : Une démarche de valorisation $\mathrm{du}$ littoral au Pays Marennes-Oléron
}

\author{
Jean-Baptiste Bonnin, Yannick Bruxelle et Christophe Pelaprat
}

1 Conscients de leurs spécificités insulaires (entre autres la pression touristique, le vieillissement de la population) et de la fragilité de leur milieu de vie, les élus et habitants se sont mobilisés autour d'un projet de développement durable sur le territoire du Pays Marennes-Oléron. Ils proclament leur engagement dans une stratégie d'affirmation de l'identité de ce territoire. L'association IODDE (île d'oléron, Développement Durable, Environnement) au cœur de cette démarche a souhaité questionner cette notion d'identité de territoire. Ainsi mène-t-elle un travail d'enquête de terrain auprès d'un échantillon représentatif de la population du Pays, avec le concours d'un photographe-enquêteur qui croise son regard « extérieur » à celui des personnes qu'il interroge.

2 Cet article, après avoir détaillé le contexte spécifique oléronais, fera état des premiers résultats de ce travail en cours. Il envisagera les ambitions éducatives de ce projet et les effets escomptés sur le territoire, montrant la place que peuvent prendre dans de telles démarches des associations concernées à la fois par la mise en route du développement durable et par l'éducation qui doit l'accompagner.

\section{Un territoire préoccupé par son identité}

\section{Un contexte bien particulier}

Soumis à une pression anthropique en très forte croissance depuis quelques décennies, le littoral charentais, et en particulier l'île d'oléron, a subi d'importantes modifications. Elles sont d'ordre quantitatif: avec 22000 habitants l'hiver, l'île accueille jusqu'à 300000 personnes simultanément en été, ce qui entraîne des conséquences sur les infrastructures et l'organisation de la vie locale. Elles sont aussi d'ordre qualitatif: 
entre autres l'afflux de nouveaux habitants sur le littoral (dont beaucoup de retraités) génère de nouveaux besoins; le tourisme de masse répond également à certains concepts normés. Il en résulte une transformation du fonctionnement du territoire, temporaire à l'échelle d'un cycle annuel (les contrastes d'ambiance sont très forts entre les saisons estivale et hivernale), mais aussi profonde et durable.

En particulier, le Pays $^{1}$, depuis plusieurs années, ne peut que constater un vieillissement de sa population, par exemple dans les quelques situations typiques suivantes :

- Des personnes ou couples qui, après être venus sur l'île d'Oléron passer des vacances (parfois depuis tout jeune : l'île a une grande tradition d'accueil de colonies de vacances ${ }^{2}$ de bord de mer) ont, une fois retraités, augmenté leur temps de séjour, acquis une résidence secondaire ou se sont établis définitivement.

- Le manque de terrains disponibles fait que, par le simple jeu de la concurrence foncière et du coût de l'habitat, les jeunes ménages actifs, a priori moins aisés financièrement que la population citée précédemment, peinent à s'installer. Seuls les enfants de familles locales possédant des terrains (ou en ayant hérité) ou non locales, mais dans des situations relativement fortunées, ce qui reste rare, pourraient trouver de quoi se loger sur l'île.

- L'habitat étant globalement plus abordable sur le continent, des jeunes qui trouvent un emploi sur Oléron préfèrent demeurer sur la côte charentaise, à Marennes par exemple. C'est d'ailleurs aussi le cas de certains oléronais de souche contraints de renoncer à vivre sur l'île : certains partent acquérir une expérience professionnelle puis reviennent sur oléron qui garde un fort pouvoir attractif.

\section{Un territoire riche de bonnes intentions, de bonnes volontés, mais n'échappant pas aux blocages}

Dans leurs travaux à l'échelle du Pays Marennes-Oléron, comme lors de la rédaction de la Charte de développement durable (2004) et du Schéma de Cohérence territoriale (SCoT, 2005), les habitants et les élus ont souhaité à deux reprises " affirmer l'identité du territoire » en tant qu'axe stratégique numéro un. Dans leur motivation, on peut percevoir une certaine crainte de la banalisation, notamment paysagère. Le développement des zones artisanales, des enseignes commerciales, de certains aménagements de voirie, répondent à certains standards et grignotent le territoire. Ce type de banalisation, ressentie comme une agression esthétique, pourrait être facteur de désintérêt du territoire, pour les habitants et les visiteurs, et finalement d'un certain « laisser-aller " pouvant générer une dynamique de cercle vicieux.

6 Si l'enjeu semble relativement partagé parmi les acteurs locaux, les gestes concrets manquent encore. Pourquoi ? D'une part, les réflexes d'aménagement inscrits dans une certaine perspective économique sont prégnants. D’autre part, cette «identité du territoire » n'a pas fait l'objet à ce jour d'une véritable définition. Il s'agit pourtant, à une échelle individuelle, d'une véritable corde sensible des habitants, en réaction à une transformation rapide et subie de leur environnement. Certains vont jusqu'à «fuir » leur île natale en été face à l'affluence touristique, montrant ainsi leurs limites à endurer les modifications de leur environnement.

Un premier point de blocage a été vite identifié : les travaux structurants à l'échelle du Pays Marennes-Oléron (Charte, SCoT) n'ont pas pu cerner « une » identité de ce Pays. L'identité insulaire est notamment particulière. Notons qu'à l'observation, à l'intérieur 
même de l'île, on peut découvrir des différences importantes, entre un nord plutôt agricole et viticole, un sud à vocation balnéaire séculaire, une côte occidentale orientée vers l'océan et la pêche avec le port de La Cotinière, ou encore un sud-est tourné vers le bassin ostréicole. Ainsi pouvons-nous penser que la piste menant à la définition d'une identité unique au Pays Marennes-Oléron n'était probablement pas la bonne et qu'elle mérite d'être interrogée.

\section{Des associations au cœur des acteurs du territoire}

\section{Des associations soucieuses de développement durable}

8 En France, les associations jouent depuis longtemps un rôle important dans les territoires, rôle qui s'est trouvé légalement renforcé depuis le Grenelle de l'Environnement ${ }^{3}$ et le Rapport Pancher ${ }^{4}$ sur «la gouvernance à cinq ». Elles constituent une force reconnue de la société civile. Dans le cas qui nous concerne, elles sont structurées à différentes échelles et fonctionnent en dynamique de réseau.

9 Ainsi au plus près du terrain, l'association IODDE s'est créée dans le prolongement du Conseil de développement pour justement mettre en œuvre des actions concrètes sur les objectifs dégagés par la charte (principale production dudit Conseil). Elle s'est attachée à rassembler en interne plusieurs acteurs du développement durable. Elle travaille volontiers sur un mode participatif et en coopération (complémentarité même) avec les institutionnels. Il semble que le défi d'opérer dans une perspective militante tout en s'appuyant sur des bases scientifiques (transparence de la méthode) et partagées ait été réussi dans un précédent travail ${ }^{5}$, ce qui apporte une certaine légitimité à l'association, reconnue par les autorités du Pays et ses partenaires qui ont attribué des fonds à IODDE pour le travail présenté ici.

\section{Des associations ayant des ambitions éducatives}

\section{Avec une éthique affirmée}

10 Fortement préoccupée par les questions liées à l'éducation de tous publics, IODDE coopère avec le réseau du GRAINE Poitou-Charentes dont elle est membre, et est en lien avec le réseau national École et Nature. Toutes ces associations ont réfléchi sur leur éthique (voir par exemple la « charte de qualité de l'éducation environnementale $»^{6} \mathrm{du}$ GRAINE Poitou-Charentes) en lien avec leurs pratiques. Elles appliquent les principes du développement durable, notamment en raisonnant à long terme. La co-construction est l'un de leurs modes d'agir. Dans le cas présent, confrontées à une pluralité de points de vue et d'intentions, il leur est indispensable de s'appuyer sur une structure de projet très fiable, des valeurs aux moyens en passant par les différents niveaux d'objectifs.

\section{Des convictions éducatives}

11 Nos associations ont à éclaircir des malentendus qui tendent à les enfermer dans des caricatures, par exemple : l'éducation est exclusivement l'affaire de l'école, l'éducation est destinée seulement aux enfants, l'éducation ce n'est pas de la culture ou encore, l'environnement, c'est pour les «écolos». Nous revendiquons clairement une éducation " pour tous, partout et tout au long de la vie $»^{7}$, qu'elle se pratique dans des 
cadres formels ou pendant des temps de loisirs. Apprendre tout au long de la vie ou encore, comme le dit joliment Hélène Trocmé-Fabre (1999), " habiter en apprenance ", n'est-ce pas le plus beau des projets et un très grand plaisir que de ressentir la joie d'apprendre et de découvrir que l'on a appris?

12 Au niveau d'un territoire à habiter, thématique de ce colloque, nous suivons Lucie Sauvé lorsqu'elle dit :

L'éducation relative à l'environnement peut contribuer au renforcement d'une affirmation de soi-même, individuellement et collectivement, ici et maintenant, en lien avec l'appartenance au lieu comme condition essentielle de l'exercice d'une responsabilité environnementale. (Sauvé, 2009, p. 4)

13 Tout en pensant qu'il nous faut faire le lien avec notre appartenance au monde. Car, ce petit bout de Terre où nous vivons doit servir à une appréhension globale du monde. Nous adhérons aussi aux propos d'Edgar Morin lorsque, interrogeant les savoirs nécessaires à une éducation contemporaine, il repère des savoirs nécessaires actuellement quasiment absents des circuits d'éducation formelle - tels que :

- Enseigner la condition humaine : interroger notre condition humaine, c'est interroger en premier notre situation dans le monde (Morin, 1999, p. 49);

- Enseigner l'identité terrienne : qui ouvrirait la voie pour civiliser la Terre conçue comme maison et comme jardin de l'humanité (Morin, 1999, p. 79).

\section{Et des « arts de faire » sur le terrain}

Cette formule empruntée à Michel de Certeau (1990) souligne bien que nous sommes avant tout des personnes de terrain en recherche de cohérence et que nos pratiques cherchent à être le reflet de notre éthique. Ainsi, s'appuyant sur une éthique particulière, les associations d'éducation à l'environnement et au développement durable sont-elles porteuses de démarches au sein desquelles peuvent se distinguer des buts éducatifs constituant des entrées, voire des étapes.

- Accueillir et apprendre à accueillir : en développant l'attention à l'Autre différent, prenant en considération ses «cris du cœur» (Majo Hansotte, 2010) et ses représentations. La reconnaissance de chacun, dans l'idée que la diversité est une richesse, est favorisée par des techniques de mise en relation permettant la mise en route d'une éthique de discussion.

- Découvrir et apprendre à découvrir : c'est-à-dire partir du terrain et du réel en permettant l'expression des réalités perçues et vécues par les personnes. Il s'agit de s'informer d'une manière la plus exhaustive possible et de faire circuler ces informations, puis d'être en capacité de reconstruire et représenter la complexité. C'est donc faire appel de façon combinée à des démarches analytiques et systémiques.

- Comprendre et apprendre à comprendre : dimension qui exige de prendre du temps afin de permettre la confrontation des différents champs de savoirs, mais aussi de respecter les rythmes des personnes et de cultiver leur curiosité, car comme le dit Edgar Morin (1994, p. 114) «comprendre c'est sans cesse apprendre et réapprendre» et s'inscrire dans une éthique de la complexité.

- Décider et apprendre à décider : en étant attentif à des processus de prises de décisions mettant les personnes en sécurité, leur laissant le temps de mûrir leur choix, faisant en sorte que toute parole, même extrême, soit respectée. Les techniques liées aux démarches participatives pour « apprendre ensemble à choisir ensemble » (Bruxelle et Hortolan, 2009) sont alors essentielles. Dans cette conception, l'éducation à l'environnement est aussi une éducation au politique. 
- Agir et apprendre à agir : parce qu'à un moment il faut décider de passer à l'action et d'avancer avec ce que l'on est et ce que l'on sait! Basculement très délicat et aléatoire dans une démarche pédagogique qui fait se poser beaucoup de questions aux éducateurs (Marleau, 2009).

Toutefois, ces éducateurs ne travaillent pas isolément et ils ont aussi défini un principe qui est celui de travailler en partenariat (compris dans une définition exigeante dépassant la simple concertation, mais développant des démarches collaboratives ou coopératives).

\section{Une démarche engagée sur le territoire Marennes- Oléron}

\section{Pour interroger cette identité}

\section{Questionner la notion même d'identité}

Il s'agit là pour nous d'oser questionner les mots et les évidences constituant les titres des documents officiels déclarant vouloir, comme nous l'avons déjà dit, "affirmer l'identité du territoire ». Or, comme on a pu le voir, notamment dans une récente actualité, mais aussi comme de nombreux chercheurs comme Pierre Bourdieu ou Erving Goffman ont pu depuis longtemps l'analyser, le thème de l'identité est piégeant. Il peut revêtir certains risques de jugements de valeur sur les " autres", provoquer le développement d'un renfermement identitaire ou encore créer ces identités de toute pièce, comme le dit Anne-Marie Thiesse (1999).

Pour notre part, en accord avec les travaux de Philippe Zarifian (2009), nous pensons que cette notion, qui correspond à un monde de délimitation entre un intérieur et un extérieur, tend à opposer les personnes et les territoires (étrangers, adversaires, ennemis, etc.). Cet auteur lui préfère la notion d'appartenance qu'il voit comme « une approche modeste située en amont de toute identité [et qui] dit de nous ce qui est commun. Elle définit nos affinités - et notre appartenance première est notre appartenance à la nature ». Pour lui, l'appartenance est ce qui nous pousse et il évoque une "appartenance commune aux devenirs collectifs », c'est-à-dire des groupes de personnes qui se soudent par les devenirs dans lesquels elles sont engagées.

18 En l'occurrence, au cours de la démarche, il aura été nécessaire de rappeler fréquemment que le centre de préoccupation n'est pas de qualifier les personnes, mais bien le pays et comment il est perçu par celles-ci. Ainsi, plutôt que de parler de l'avenir d'un territoire, lui préférons-nous la notion dynamique de devenir.

Partir des réalités de terrain, avec le moins d'a priori possibles, et en acceptant la complexité

19 Un travail est mené auprès des acteurs et des populations du Pays de Marennes-Oléron afin non pas forcément de définir une identité précise et figée qui pourrait avoir un effet excluant qui n'est pas recherché, mais de valoriser cette identité par le fait même de se poser la question et de confronter ses idées. Loin de vouloir dresser un travail d'inventaire et de définir de manière exhaustive les éléments identitaires, la démarche 
proposée ici est d'identifier l'approche culturelle du territoire, la manière dont les habitants et les visiteurs le perçoivent, l'habitent, le découvrent, l'utilisent.

\section{Pour entendre les habitants}

\section{Les intentions de la méthode}

Il s'agit de recueillir la parole d'un échantillon de différentes catégories de la population, en suscitant la discussion à l'aide de supports photographiques issus d'un regard extérieur au territoire, ces derniers révélant un éventail de paysages de Marennes-Oléron. L'échantillon de personnes interrogées comprend en particulier :

- les habitants permanents : de ceux qui y sont nés aux «néo-oléronais ",

- les résidents secondaires, à la fois touristes et habitants,

- les touristes, visiteurs éphémères, mais réguliers pour certains,

- les professionnels : ceux qui font vivre le territoire et le perçoivent au travers de leur activité,

- les acteurs publics : élus et professionnels d'organismes publics, associations.

Des entretiens individuels ont été mis en place avec une cinquantaine de personnes réparties selon ces différentes catégories, jusqu'à ce que nous estimions la diversité des expressions suffisantes pour le cadre de l'enquête. Ces entretiens ont duré chacun entre une heure et quatre heures. La question de l'identité territoriale n'est pas posée directement. Il est demandé aux personnes interrogées de livrer leurs regards, leurs perceptions, leurs ressentis devant différents aspects de leur territoire de vie, de loisir, de travail, etc.

\section{Le filtre du paysage et le support de l'image}

Expression du territoire vu, le paysage est l'interface entre notre perception d'un espace et ses composantes naturelles et culturelles. Il permet de révéler les différentes « couches » d'un territoire, et d'en disséquer le fonctionnement. Le paysage sera utilisé ici comme support de discussion : il permettra d'aborder la problématique de l'identité au travers d'éléments visuels très concrets et de manière transversale. Le choix du paysage, en suscitant la parole sur des réalités de terrain, permet aussi d'éviter toute approche purement identitaire dans un sens figé et exclusif. Enfin, l'expression à partir $\mathrm{du}$ paysage facilite les passerelles vers différentes thématiques: patrimoine, biodiversité, aménagement, urbanisme, tourisme, etc. Elle a la liberté de recouvrir aussi bien les visions géographique, naturaliste ou encore imaginaire de cet espace pourtant bien réel.

La photographie exprime le regard porté sur un territoire. Elle permettra ici de confronter un regard "découvreur", extérieur à ceux de personnes vivant sur le territoire, habitants ou visiteurs. L'image a aussi l'avantage d'apporter un support de discussion accessible à tous, notamment en associant les paroles aux images. C'est enfin un média facile à transmettre et à réutiliser, pouvant être valorisé sous différentes formes. 


\section{Pour essayer de comprendre : premiers résultats}

\section{Des premiers regards...} aux premiers interlocuteurs interrogés lors de la première série d'entretiens, en avril 2010 - la deuxième série se déroulant fin juin / début juillet. Les supports photographiques (figures 1 et 2) de discussion couvrent l'ensemble du territoire et expriment un regard de simple observateur des caractéristiques du paysage. Seule entorse à la neutralité, les images sont présentées en diptyques, révélant ainsi des problématiques, exprimant des contrastes, des similitudes. L'utilisation des photographies a permis d'alimenter la discussion sans la brider, les personnes interrogées n'y faisant pas forcément référence. Les images peuvent permettre de relancer le débat et de balayer l'ensemble des problématiques du territoire.

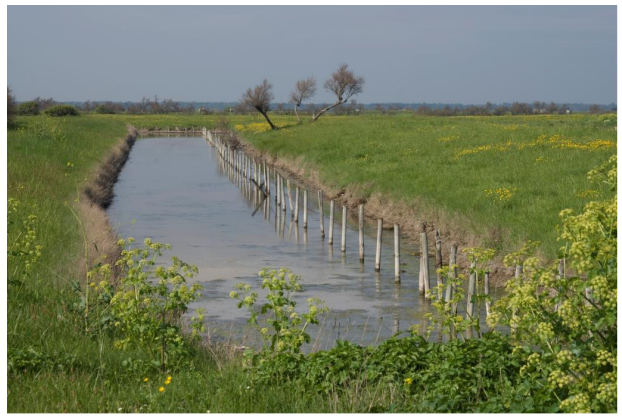

St Pierre La Vieille Perrotine

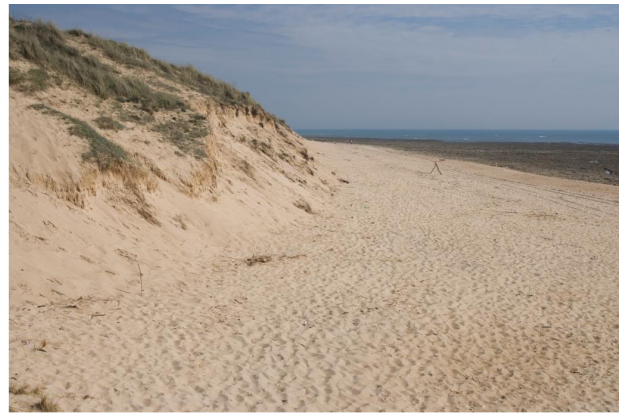

St Pierre Les Sables

Figure 1 : Exemples de supports photographiques - Entre terre et mer

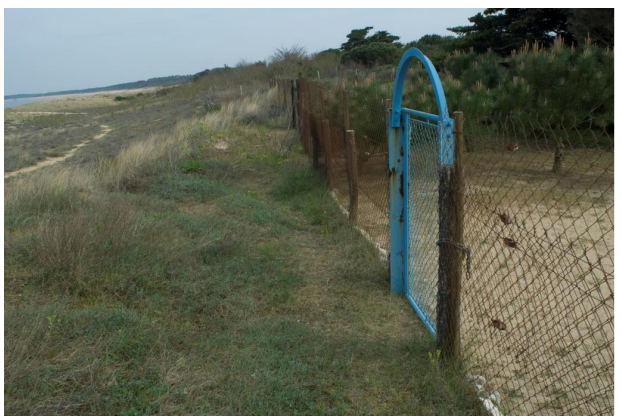

Le Douhet

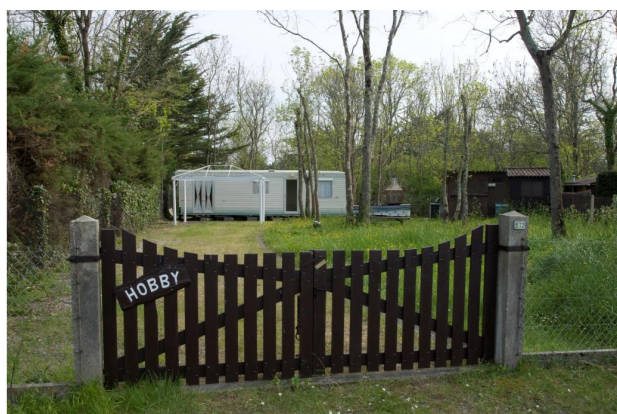

St Georges d'Oléron

Figure 2 : Exemples de supports photographiques - Espace et tourisme purement identitaire, et celui d'un inventaire patrimonial à la Prévert.

\section{... Aux premières paroles}

Des thématiques, des jalons patrimoniaux, des enjeux, etc.: les premiers entretiens permettent déjà de repérer quelques éléments de perception du territoire. 
Quelques constats d'ordre global :

- Les éléments qui semblent être des repères identitaires évidents (phare de Chassiron, citadelle du Château) sont à peine abordés, et suscitent peu de commentaires.

- L'accent est mis sur la manière d'utiliser son terroir, d'user de son territoire : la pratique des marais, la pêche à pied, l'entretien des écluses à poissons ${ }^{8}$, etc.

- L'omniprésence du tourisme, la brutalité des phénomènes naturels sont systématiquement abordées, ces thèmes font partie de la vie quotidienne du territoire, de même que les activités économiques (pêche, ostréiculture) et la prolifération de l'habitat.

- Bien sûr, l'île et le continent restent deux entités distinctes et ressenties telles quelles : on est de l'une ou de l'autre, sans toutefois les opposer, plutôt en soulignant leur complémentarité. lieu de vie s'expliquant au travers de ce que l'on en fait. Un premier constat plutôt positif pour une démarche qui entend éviter le piège identitaire et vise des finalités concrètes en terme de développement durable et d'éducation à l'environnement.

\section{Bilan d'étape : vers un territoire « actif » prenant en main son devenir}

\section{Recul sur la méthode utilisée dans cette enquête}

En l'occurrence, même si les efforts d'explication sont faits, il reste plusieurs interrogations: comment l'interlocuteur ressent-il nos questions? Quel sera son objectif en nous répondant? Montrer qu'il connaît bien son territoire? Mieux que le voisin? Que son territoire est le plus merveilleux? Avoir au contraire une approche critique pour tenter de mettre en évidence certains problèmes? Un premier biais peut 
donc entrer en jeu dès les premiers contacts. Notons toutefois que cette enquête, diagnostic de départ, recherche d'interprétation avant une mise en dynamique souhaitée vers un territoire "actif», n'a pas la prétention d'être une recherche s'inscrivant dans la rigueur d'un cadre positiviste.

Les prémices de cette action ont également montré à quel point ce thème était "politique». Cela s'illustre, par exemple, par le fait que dès qu'il s'est agi de commencer à travailler avec les administrateurs de l'association IODDE, un engouement certain s'est manifesté pour répondre à l'enquête. Cette motivation n'était peut-être pas innocente : chacun voulait sans doute se raconter, donner sa vision du territoire, mais aussi peut-être exprimer ses attentes, dans la droite ligne de son engagement associatif. Il peut tout à fait en être de même pour les autres interlocuteurs, chacun impliqué à sa façon dans la vie locale. Ainsi, il faut veiller à faire la part des choses au moment des entretiens et de leur analyse.

L'approche du paysage par la photo, même si elle est utilisée ici comme un support et non comme un carcan, oriente inévitablement la nature des réponses, en termes de contenus. Des pans entiers de l'identité locale peuvent ne pas être recueillis par cette méthode. L'odeur des immortelles sur les dunes au crépuscule, les "conversations " permanentes des oies bernaches en hiver, les premières cuisines de seiches du printemps, mais aussi des aspects disparus, mais pourtant bien caractéristiques pourraient être oubliées, donnant une image tronquée, très " matérielle ", de l'identité. Charge aux maîtres d'œuvre de ne pas viser une définition exhaustive des caractéristiques identitaires sous peine d'être finalement infidèle aux propos de chacun

Or, le propos est bien au contraire d'arriver à un socle de représentations qui puisse recueillir une adhésion large d'acteurs et aboutir à une volonté de valorisation des caractéristiques identitaires. Ces biais « humains » étant posés et conscientisés, il s'agit donc de promouvoir une image d'honnêteté et de rigueur dans la démarche, qui permettra d'enclencher une dynamique de même nature chez les interlocuteurs. Cette posture doit permettre de construire ensemble, même avec des intentions différentes, sur au moins des fondements communs et reconnus par tous.

À l'usage, on peut se rendre compte que la méthode retenue n'est pas restrictive. Elle permet effectivement d'engager la discussion, mais ne la guide pas vers un résultat préétabli. Tout au long de la démarche, il a été rappelé autant que nécessaire que l'idée du projet n'était pas d'inventer une nouvelle manière de faire passer un certain nombre d'idées préexistantes, mais bien d'obtenir une image la plus fidèle possible du ressenti des interlocuteurs. Le fait de confier le choix de la méthode d'entretiens, leur conduite et leur analyse, vise à limiter autant que possible les choix arbitraires dans les questions et les éléments retenus. La multiplication des entretiens et la diversité des profils des personnes interrogées permettent également de lisser un certain nombre de biais.

\section{Développer le « sens » du territoire ou « La leçon des huîtres »}

S'il ne venait à l'idée de quiconque de commander des huitres de Bouzigues ${ }^{10}$ dans un restaurant de Marennes-Oléron, combien savent qu'en réalité, dans la plupart de ces établissements (environ 90 \% selon la Maison du tourisme de l'île d'oléron et du Bassin de Marennes), le poisson qui suit dans le menu a été généralement pêché par des bateaux industriels en Atlantique Nord, et suivi les différentes étapes commerciales via 
le marché de Rungis, et donc a voyagé des centaines de kilomètres et dans certains cas séjourné en congélation, et ce, même sur les terrasses qui proposent la vue sur le port de la Cotinière, réputé pour son dynamisme et l'excellente qualité de ses apports en produits de la mer qui sont, eux, en grande partie exportés dans ces mêmes circuits !

Il faut donc, et c'est presque un comble dans l'absolu, encore lutter pour inciter les acteurs économiques, en particulier la filière alimentaire, à se fournir localement. Si les huîtres ont gagné ce privilège, c'est au prix d'efforts de communication importants, et à connotation très identitaire: en témoigne le slogan développé par le syndicat conchylicole: "l'huître a un pays, Marennes-Oléron ». La réussite de cette communication est de capitaliser sur l'omniprésence des éléments dans le paysage : parcs et claires à huîtres, cabanes et bateaux ostréicoles, tas de coquilles et matériels divers, et bien sûr dans chaque marché.

Au-delà des aspects économiques, c'est la notion de « sens » qui apparaît là. Cela a du sens de manger des huîtres ici, de s'intéresser à leur mode de production, à la vie des gens qui les élèvent et au territoire qui permet leur croissance, grâce à l'interface de l'eau salée et des apports d'eaux douces des fleuves côtiers. Cela a du sens de préserver la qualité de l'environnement, à la base de cette qualité de vie. Ne serait-ce pas justement ce sens qui produit un effet d'harmonie, qui produit le bien-être, la fierté d'être un acteur d'un territoire, et par extension la volonté d'en préserver les qualités ? L'enjeu pour le territoire est peut-être de reconquérir ce sens. Reconquérir, c'est d'une part rompre avec la logique d'aménagement passif (selon une compilation d'intérêts particuliers de court terme) et d'autre part capitaliser sur les éléments réellement identitaires.

Cette valorisation du territoire a potentiellement des implications environnementales très directes. Par exemple, la mise en valeur de produits locaux (démarche de circuits courts, à laquelle de nombreux territoires réfléchissent y compris Marennes-Oléron) évite des déplacements générateurs d'effet de serre. Elle permet aussi une économie mieux maîtrisée et moins dépendante des "grandes tendances » dont on connaît les effets banalisants. Dans ce domaine, beaucoup reste à faire.

Sur le plan du développement local, la volonté est également de produire des outils d'aide à la décision. Ce travail pourra servir de base à d'autres réflexions territoriales : schémas d'urbanisme, politiques environnementales, vie culturelle et sociale, promotion touristique, etc. Ces «outils " peuvent être directement des contenus, à partir des connaissances recueillies par les enquêtes : éléments importants à préserver ou à valoriser, écueils à éviter en termes d'aménagement du territoire. Il peut également s'agir d'éléments méthodologiques: faire participer, respecter une démarche scientifique et transparente.

\section{Contribuer à l'évolution d'un territoire}

41 Cette action, toujours en cours, illustre comment une initiative associative peut contribuer à l'évolution d'un territoire :

- Par une recherche interprétative: sur la base d'une méthodologie scientifique, l'investigation et la concertation auront permis d'acquérir des compléments de connaissance, non seulement sur le terrain d'étude, mais également sur la perception qu'en ont les habitants, au travers de leurs constats, propositions et interrogations.

- Par l'accompagnement d'un territoire, en produisant du lien et du débat permanent entre les acteurs, et en réinvestissant les enseignements de ces échanges dans les programmes 
structurants du pays : il peut s'agir d'atouts ou de menaces à prendre en compte, d'objectifs à définir pour aller vers cette affirmation de l'identité qui était apparue dès la création du pays comme une évidence.

- Par l'éducation, intégrée dès la prise d'initiative comme objectif transversal, décliné selon les différentes étapes de la démarche.

Il apparaît également transversal de respecter cette quête personnelle, ce sentiment viscéral d'appartenance à un territoire ce que Raymond Depardon (2000, p. 42) nomme «la quête d'un lieu acceptable, recherche d'un endroit pour vivre qui soulève la question "Qu'est-ce que je fais là ? " ", cette recherche afin de se sentir bien sur Terre, se sentir chez soi. Ainsi, faire l'effort de comprendre son territoire constitue un premier pas pour le rendre actif, s'y intéresser, s'y impliquer, s'y sentir bien et l'aimer, le partager et le préserver. C'est donc bien le cœur de cette dynamique actuelle: partager le territoire tout en le préservant. La difficulté est particulièrement importante dans l'une des stations les plus touristiques de France ${ }^{11}$. Même si ce projet aura permis d'ouvrir quelques voies pour une meilleure prise en compte des enjeux identitaires du pays Marennes-Oléron, il ne constitue qu'une étape stratégique pour emmener les acteurs aussi loin que possible dans une démarche de reconquête : une reconquête de contenus, de sens, et d'outils de maîtrise d'avenir.

\section{BIBLIOGRAPHIE}

Bruxelle, Y. et Hortolan, M. (2009). Entre morale et éthique : apprendre ensemble à choisir ensemble. Éducation et Francophonie, 37(2), 44-62. Revue en ligne : www.acelf.ca

De Certeau, M. (1990). L'invention du quotidien. Arts de faire. Paris : Folio Essais nº 146. Depardon, R. (2000). Errance. Paris : Seuil.

Hansotte, M. (2010). Pour une méthodologie des intelligences citoyennes, repères théoriques et démarche pratique de terrain. Intervention lors du Congrès du Réseau École et Nature, mars 2010, Actes de la journée de réflexion Congrès 2010.

Marleau, M.-È. (2009). Des liens à tisser entre la prise de conscience et l'action environnementales. Éducation et Francophonie, 37(2), 11-32. Revue en ligne : www.acelf.ca

Morin, E. (1999). Les sept savoirs nécessaires à l'éducation du futur. Paris : Seuil.

Sauvé, L. (2009). Vivre ensemble sur Terre : enjeux contemporains d'une éducation relative à l'environnement. Éducation et Francophonie, 37(2), 1-10. Revue en ligne : www.acelf.ca

Syndicat Mixte du Pays Marennes Oléron (2004). Charte de développement durable du Pays Marennes Oléron. Saint-Pierre d'Oléron : Syndicat Mixte du Pays Marennes Oléron.

Syndicat Mixte du Pays Marennes Oléron (2005). Schéma de cohérence territoriale du Pays Marennes Oléron. Saint-Pierre d'Oléron : Syndicat Mixte du Pays Marennes Oléron.

Trocmé-Fabre, H. (1999). Réinventer le métier d'apprendre. Paris : Éditions d'Organisation. Zarifian, P. (2009). L'émergence du peuple monde. Paris : PUF. 


\section{NOTES}

1. Dans le sens utilisé ici, les «Pays » sont des territoires formalisés par la Loi d'Orientation et d'Aménagement pour le Développement Durable du territoire (LOADDT ou encore Loi Voynet). L'esprit de cette loi était de fournir à ces échelles géographiques, cohérentes de par leur identité commune, des capacités d'organisation et de projet. Les pays sont à une échelle intermédiaire entre les communautés de communes et les départements. Le Pays Marennes-Oléron regroupe deux communautés de communes : celle du Bassin de Marennes (sept communes) et celle de l'île d'Oléron (huit communes). La structure juridique est un syndicat mixte, fondé sur sa charte de développement durable. Le Pays anime plusieurs démarches territoriales (programmes européens, touristiques, analyses socio-économiques, etc.).

2. On parle maintenant de centres de loisirs, mais le terme de colonies de vacances est resté, notamment s'agissant de personnes qui les ont fréquentées à l'époque. Les plans et schémas locaux d'urbanisme utilisent d'ailleurs encore cette dernière terminologie.

3. Le «Grenelle de l'environnement » est un programme d'envergure en France qui a mobilisé depuis 2008 différents experts et représentants de militants, dans le but de mettre en place des règlements et mesures environnementales.

4. Le Rapport de juillet 2008 peut être consulté sur Internet: http://www.assembleenationale.fr/13/rapports/r1774.asp

5. L'association IODDE a initié dès 2004 un travail d'ampleur significative sur la problématique de la pêche à pied récréative, comprenant un volet scientifique (diagnostic rigoureux de la pression de pêche, impacts sur les écosystèmes), un volet pédagogique (production d'outils, formation de relais, opérations de terrain, etc.) et une forte dimension de concertation des acteurs. La démarche a été plébiscitée localement et a obtenu différentes reconnaissances nationales (prix environnement 2008, coups de cœur de Fondations nationales, etc.). Aujourd'hui, la façon de travailler expérimentée sur Marennes-Oléron est celle qui est retenue pour le programme national sur la pêche à pied récréative (2011-2015) mis en place par l'Agence des Aires Marines Protégées et le Conservatoire du littoral.

6. Voir le site : www.grainepc.org

7. Voir la Lettre du GRAINE Poitou-Charentes $n^{\circ} 18$ : www.grainepc.org

8. Les écluses à poissons sont des pièges construits en murs de pierres sèches sur l'estran (zone de balancement des marées). À marée descendante, le poisson y reste emprisonné. Des équipes de détenteurs s'occupent de l'entretien de ces murets, et se répartissent les marées de pêche. Existant depuis le Moyen Âge, les écluses ont été plus de 200 sur le tour de l'île puis ont décliné. Aujourd'hui, dix-sept écluses y sont encore en activité.

9. La tempête Xynthia a frappé toute la région le 28 février 2010 en pleine nuit. Provoquant une submersion marine dans de nombreux villages du pays, le phénomène a des effets durables sur l'impression de vulnérabilité des habitants.

10. Ce sont des huîtres cultivées dans le sud de la France (Étang de Thau).

11. La Charente-Maritime est depuis 4 ans le deuxième département touristique de France après le Var. Bien entendu, cette pression s'exerce en priorité sur les côtes et encore plus fortement sur les îles, dont la plus grande est Oléron. 


\section{RÉSUMÉS}

Ces dernières décennies, les côtes atlantiques métropolitaines sont des plus attractives. Cette grande tendance se traduit par l'accroissement rapide de la population littorale, qu'elle soit résidente ou touristique. Certains habitants de Marennes-Oléron ont mis en évidence que cet afflux de nouveaux venus risquait de mener à une certaine banalisation de leur territoire. En réaction, dès les années 2003 à 2004, le Pays Marennes-Oléron a inscrit dans ses principaux schémas de développement la volonté d'« affirmer l'identité du territoire ». Cependant, cette «identité » n'a pas encore fait l'objet d'une véritable définition, ce qui réduit les chances de produire des effets à cette volonté locale. En contribution, l'association IODDE (développement durable local) s'est associée avec l'Agence Biljara (géographie, démarches de territoires) et le GRAINE Poitou-Charentes (éducation à l'environnement) pour réaliser un travail d'enquête et de capitalisation des perceptions d'habitants ou d'usagers sur ce qui pourrait être une base partagée de ces caractéristiques, et engager une réflexion sur sa valorisation.

For the last few decades, the metropolitan areas on the atlantic coasts have become more attractive, which is shown by the rapid increase of the littoral population, whether residents or tourists. For some inhabitants of Marennes-Oléron, this influx of newcomers threatened to make their territory humdrum. In reaction, by 2003, the "Pays Marennes-Oléron ", in its development planning, set the goal of " asserting the identity of the territory". However, this "identity " has not been defined yet, which reduces the chances to produce the desired local effects. In contribution, the association IODDE (local sustainable development) joined with the agency Biljara (geography, initiatives of territories) and the association GRAINE Poitou-Charentes (environmental education) to develop a research project and to capitalize on the perceptions of inhabitants or users of this territory on what could be a shared vision of its characteristics and what could be built from that.

\section{AUTEURS}

\section{JEAN-BAPTISTE BONNIN}

Jean-Baptiste Bonnin est coordinateur de l'association IODDE - Île d'oléron Développement Durable Environnement / CPIE Marennes-Oléron. Cette structure acteur de terrain composée majoritairement d'habitants, s'implique dans de nombreux aspects du développement local, en prenant des initiatives ou en accompagnant les différents acteurs vers une réflexion et une mise en pratique des principes du développement durable. Animateur et rédacteur de plusieurs Agendas 21 locaux participatifs (Oléron, Romans-sur-Isère, La Rochelle) et membre de l'Union des CPIE, il est particulièrement intéressé par l'implication des habitants sur la vie de leur territoire.

\section{YANNICK BRUXELLE}

Retraitée de l'Éducation nationale, engagée de longue date dans les réseaux associatifs d'éducation relative à l'environnement et ayant mené des travaux de recherche en sciences de l'éducation, Yannick Bruxelle est maintenant bénévole au GRAINE Poitou-Charentes.

\section{CHRISTOPHE PELAPRAT}

Géographe de formation, Christophe Pelaprat est photographe et rédacteur indépendant (Agence Biljara). 